\title{
The analysis of instability and strain concentration during superplastic deformation
}

\author{
Li Chuan Chung, Jung-Ho Cheng * \\ Department of Mechanical Engineering, National Taiwan University, No. 1, Sec.4, Roosevelt Rd., Taipei 106, Taiwan
}

Received 4 April 2000; received in revised form 22 November 2000

\begin{abstract}
The instability and the localization process of unstable plastic flow during constant pressure bulging of superplastic Ti-6Al-4V sheets at $900^{\circ} \mathrm{C}$ are analyzed. A generalized stability criterion is developed by generalizing Hart's stability criterion [Acta Metall., 15 (1967) 351] in terms of strain and strain rate. According to the stability criterion, a new concept of the 'flow localization factor', which enables a quantitative description of the localization process of unstable plastic flow, is presented. A finite element model that simulates experiments on constant pressure bulging is applied to compute the flow localization factor at the fracture point. It is found that the localization process of unstable plastic flow during constant pressure bulging can be divided into three stages: (1) the developing period of initial localized flow, (2) the steady stage of strain concentration, and (3) the accelerating stage of strain concentration, which results in the final fracture. (C) 2001 Elsevier Science B.V. All rights reserved.
\end{abstract}

Keywords: Finite element model; Flow localization factor; Instability; Stability criterion; Strain concentration; Superplasticity; Unstable plastic flow

\section{Introduction}

Superplasticity in materials is characterized by large amounts of uniform deformation, and interest in superplastic forming (SPF) has been considerable. To take advantage of SPF, improve productivity, and at the same time avoid fracturing during the forming process, further understanding of the fracture mechanisms of superplastic deformation is required, where instability is one of the most significant factors [2-4].

The failure of superplastic sheet metals is a result of the combination of, and interaction between, two processes: unstable plastic flow and internal cavity evolution [5]. It has been shown by Zhou et al. [6] that for materials that are less sensitive to cavity growth, the fracture mode is dominated by unstable plastic flow.

The instability of superplastic deformation has been the subject of several studies. By combining Hart's stability criterion [1] and a microstructure-based constitutive equation, a variable strain-rate deformation path

\footnotetext{
* Corresponding author. Fax: + 886-2-23631755.

E-mail addresses: r1852203@w2.me.ntu.edu.tw (L.C. Chung), jhcheng@w3.me.ntu.edu.tw (J.-H. Cheng).
}

that ensured a stable deformation was obtained by Ding et al. [7,8]. Du et al. [9] suggested a thermo-viscoplastic damage-instability model that was the combination of Hill's yield equation and the modified Gurson constitutive relation for porous ductile materials [10]. Taking the occurrence of localized instability or the cavity volume fraction's reaching a critical value as a fracture criterion, the forming limit was predicted.

The existing approaches are only able to predict the instability of materials or simply to assume that forming limits are reached as long as instability occurs. However, it is too conservative to take instability as the forming limit of superplastic materials; therefore, a method that can be applied more generally to predict instability and describe strain concentration beyond instability is required. Cáceres et al. [11] proposed an instability parameter to describe the development of instability under uniaxial tension. However, it lacks a clear physical meaning and cannot be applied to biaxial stretching conditions. The purpose of the present work is to propose a parameter that has a physical meaning and can be applied more generally to describe the strain concentration beyond instability. 


\section{Stability analysis}

\subsection{Hart's stability criterion [1]}

Hart developed a tensile stability criterion for materials that exhibited both strain hardening and strain-rate hardening. The criterion states that the deformation is stable if, in the course of the deformation, the magnitude of the cross-section difference does not increase, or

$\frac{\delta \dot{A}}{\delta A} \leq 0$

where $A$ is the cross-sectional area, and the operator, $\delta$, signifies a variation between the nominal and a local non-uniformity. Defining the strain-hardening coefficient $(\gamma)$ and the strain-rate sensitivity index $(m)$ to be

$\gamma=\frac{1}{\sigma}\left(\frac{\partial \sigma}{\partial \varepsilon}\right)$, and $m=\frac{\dot{\varepsilon}}{\sigma}\left(\frac{\partial \sigma}{\partial \dot{\varepsilon}}\right)$

Eq. (1) can be expressed as

$\gamma+m \geq 1$.

\subsection{Generalizing Hart's stability criterion}

Hart's stability criterion applies only to uniaxial tension and 1-D plane strain conditions. In this paper, it is generalized in terms of strain and strain rate, and then, according to the generalization, the flow localization factor can be defined for both uniaxial tension and biaxial stretching. The starting point of our generalization is the equation from Ref. [1],

$\delta \dot{\varepsilon}=-\frac{\delta \dot{A}}{A}+\frac{\dot{A}}{A}\left(\frac{\delta A}{A}\right)$

which can be expressed as

$A \frac{\delta \dot{\varepsilon}}{\delta A}=-\frac{\delta \dot{A}}{\delta A}+\frac{\dot{A}}{A}$.

Combining Eq. (5) with Hart's stability criterion, Eq. (1), it can be deduced that

$\frac{\dot{A}}{A}-A \frac{\delta \dot{\varepsilon}}{\delta A} \leq 0$.

For $\delta A<0$, it becomes

$\delta \dot{\varepsilon} \leq \frac{\delta A}{A} \frac{\dot{A}}{A}$.

Since $\dot{\varepsilon}=-\frac{\dot{A}}{A}$, and $\delta \varepsilon=-\frac{\delta A}{A}$, the above criterion can be expressed as

$\delta \dot{\varepsilon} \leq \dot{\varepsilon} \delta \varepsilon$
Let $\delta x$ be the distance along the tensile axis between cross-sections $A$ and $A+\delta A(\delta x>0)$. Dividing the above equation by $\delta x$ gives

$\frac{\delta \dot{\varepsilon}}{\delta x} \leq \dot{\varepsilon} \frac{\delta \varepsilon}{\delta x}$,

or

$\frac{\partial \dot{\varepsilon}}{\partial x} \leq \dot{\varepsilon} \frac{\partial \varepsilon}{\partial x}$.

In general, the uniaxial strain and strain rate in this criterion should be replaced by the effective strain, $\bar{\varepsilon}$, and the effective strain rate, $\overline{\dot{\varepsilon}}$, respectively; the partial differential operation should be replaced by the gradient operation. Thus, we obtain our stability criterion in terms of effective strain and effective strain rate as

$\|\nabla \overline{\tilde{\varepsilon}}\| \leq \overline{\dot{\varepsilon}}\|\nabla \bar{\varepsilon}\|$.

\subsection{Flow localization factor}

After instability occurs, the process of flow localization controls the amounts of useful deformation that can be imposed prior to failure. The more serious the localization of unstable plastic flow, the closer the material is to its forming limit. The discussion in this section is to derive a parameter that characterizes the degree of flow localization, which we name the 'flow localization factor.'

\subsubsection{Uniaxial tension}

For uniaxial tension, the flow localization factor, $\xi_{\mathrm{I}}$, where the subscript ' $I$ ' indicates 'uniaxial', can be defined according to Eq. (8) as

$\xi_{\mathrm{I}}= \begin{cases}\frac{\delta \dot{\varepsilon}-\dot{\varepsilon} \delta \varepsilon}{\dot{\varepsilon} \delta \varepsilon}=\frac{\delta \dot{\varepsilon}}{\dot{\varepsilon} \delta \varepsilon}-1 & \text { if } \frac{\delta \dot{\varepsilon}-\dot{\varepsilon} \delta \varepsilon}{\dot{\varepsilon} \delta \varepsilon}>0 \\ 0 & \text { if } \frac{\delta \dot{\varepsilon}-\dot{\varepsilon} \delta \varepsilon}{\dot{\varepsilon} \delta \varepsilon} \leq 0\end{cases}$

Since

$\delta \sigma=\frac{\partial \sigma}{\partial \varepsilon} \delta \varepsilon+\frac{\partial \sigma}{\partial \dot{\varepsilon}} \delta \dot{\varepsilon}$,

$\delta \dot{\varepsilon}=\frac{\delta \sigma-\frac{\partial \sigma}{\partial \varepsilon} \delta \varepsilon}{\frac{\partial \sigma}{\partial \dot{\varepsilon}}}$

which, divided by $\dot{\varepsilon} \delta \varepsilon$, gives

$\frac{\delta \dot{\varepsilon}}{\dot{\varepsilon} \delta \varepsilon}=\frac{\frac{\delta \sigma}{\delta \varepsilon}-\frac{\partial \sigma}{\partial \varepsilon}}{\dot{\dot{\varepsilon}} \partial \dot{\varepsilon}}$ 
Since $\delta \sigma=-\frac{\sigma \delta A}{A}=\sigma \delta \varepsilon, \delta \sigma / \delta \varepsilon=\sigma ;$ therefore, Eq.

(14) gives

$\frac{\delta \dot{\varepsilon}}{\dot{\varepsilon} \delta \varepsilon}=\frac{1-\frac{1}{\sigma} \frac{\partial \sigma}{\partial \varepsilon}}{\frac{\dot{\varepsilon}}{\sigma} \frac{\partial \sigma}{\partial \dot{\varepsilon}}}=\frac{1-\gamma}{m}$

and the flow localization factor is then expressed as

$\xi_{\mathrm{I}}= \begin{cases}\frac{1-\gamma}{m}-1 & \text { if } 1-\gamma-m>0 \\ 0 & \text { if } 1-\gamma-m \leq 0\end{cases}$

in which the stability condition, $\xi_{\mathrm{I}}=0$, is equivalent to Hart's stability criterion, $\gamma+m \geq 1$ (Eq. (3)). The definition of the flow localization factor for uniaxial tension is the same as that of the 'instability parameter' defined by Cáceres [11] except that the former is defined to be zero when the deformation is stable. The instability parameter defined by Cáceres does not have a clear physical meaning, as the flow localization factor does, and it is only valid for uniaxial tension. Nevertheless, the flow localization factor can be generalized to biaxial stretching conditions, which will be done in Section 2.3.2.

\subsubsection{Biaxial stretching}

The localization factor for biaxial stretching is derived via the stability analysis for thin sheets under biaxial stretching studied in [8]. It is assumed that when the neck develops, it is normal to the maximum principal stress $\left(\sigma_{1}\right)$, and $\Delta \dot{\varepsilon}_{2}=0$ (see Fig. 1 [8]). Combining the assumptions with incompressibility and equilibrium equation produces the following equation:

$\sigma_{1}=\frac{\delta \sigma_{1}}{\delta \varepsilon_{1}}$

Defining the principal strain ratio as

$\rho=\frac{\varepsilon_{2}}{\varepsilon_{1}}$
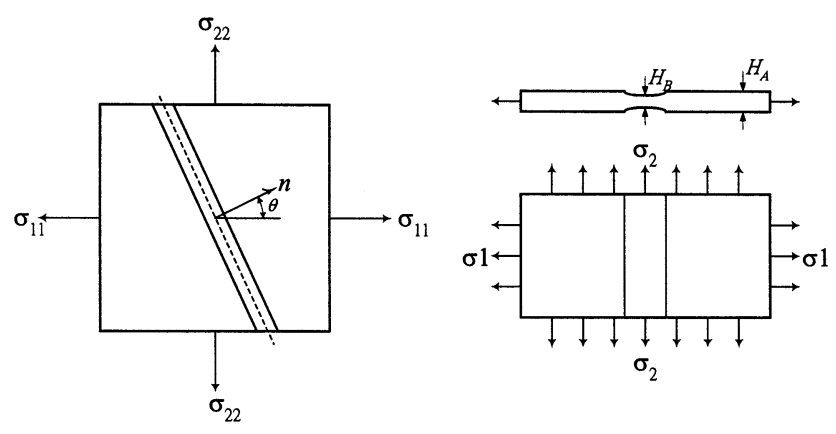

Fig. 1. Biaxial stretching of a thin sheet [8]. the equivalent strain, equivalent strain rate, and equivalent stress can be expressed as

$\bar{\varepsilon}=a \varepsilon_{1}, \overline{\dot{\varepsilon}}=a \dot{\varepsilon}_{1}$, and $\bar{\sigma}=b \sigma_{1}$

where

$a=\sqrt{\frac{4}{3}\left(1+\rho+\rho^{2}\right)}$, and $b=\frac{1}{2+\rho} \sqrt{3\left(1+\rho+\rho^{2}\right)}$.

The formulas of thin sheets under biaxial stretching presented so far were developed by Ding [8], and now, our analysis is developed based on them.

Since the neck is normal to direction 1 , and $\Delta \dot{\varepsilon}_{2}=0$, the flow localization factor under these conditions, according to our definition, should be

$\xi_{\mathrm{II}}= \begin{cases}\frac{\delta \dot{\varepsilon}_{1}-\dot{\varepsilon}_{1} \delta \varepsilon_{1}}{\dot{\varepsilon}_{1} \delta \varepsilon_{1}}=\frac{\delta \dot{\varepsilon}_{1}}{\dot{\varepsilon}_{1} \delta \varepsilon_{1}}-1 & \text { if } \frac{\delta \dot{\varepsilon}_{1}-\dot{\varepsilon}_{1} \delta \varepsilon_{1}}{\dot{\varepsilon}_{1} \delta \varepsilon_{1}}>0 \\ 0 & \text { if } \frac{\delta \dot{\varepsilon}_{1}-\dot{\varepsilon}_{1} \delta \varepsilon_{1}}{\dot{\varepsilon}_{1} \delta \varepsilon_{1}} \leq 0\end{cases}$

where the subscript II indicates 'biaxial.'

Under biaxial stretching, Eq. (14) should be modified to

$\frac{\delta \overline{\dot{\varepsilon}}}{\overline{\bar{\varepsilon}} \delta \bar{\varepsilon}}=\frac{\frac{\delta \bar{\sigma}}{\delta \bar{\varepsilon}}-\frac{\partial \bar{\sigma}}{\partial \bar{\varepsilon}}}{\overline{\dot{\varepsilon}} \frac{\partial \bar{\sigma}}{\partial \overline{\dot{\varepsilon}}}}$.

From Eqs. (17), (19) and (22), and defining

$\gamma=\frac{1}{\bar{\sigma}}\left(\frac{\partial \bar{\sigma}}{\partial \bar{\varepsilon}}\right)$, and $m=\frac{\overline{\hat{\varepsilon}}}{\overline{\bar{\sigma}}}\left(\frac{\partial \bar{\sigma}}{\partial \overline{\bar{\varepsilon}}}\right)$

we obtain the flow localization factor under biaxial stretching as

$\xi_{\mathrm{II}}= \begin{cases}\frac{1-a \gamma}{m}-1 & \text { if } 1-a \gamma-m>0 \\ 0 & \text { if } 1-a \gamma-m \leq 0\end{cases}$

Under stable conditions, $\xi_{\mathrm{II}}=0$, and Eq. (24) is reduced to the stability criterion of Ding [8], $a \gamma+m \geq 1$. Under uniaxial tension, $\rho=-0.5$, and then $a=1$, so Eq. (24) is reduced to Eq. (16). If $\rho=1$ (balanced biaxial stretching), then $a=2$; therefore, Eq. (24) becomes

$\xi_{\mathrm{II}}= \begin{cases}\frac{1-2 \gamma}{m}-1 & \text { if } 1-2 \gamma-m>0 \\ 0 & \text { if } 1-2 \gamma-m \leq 0\end{cases}$

The flow localization factor, whose physical meaning is the degree of flow localization, plays an important role in this research. If $\xi>0$ at some point during deformation, flow localization will occur at that point until the 


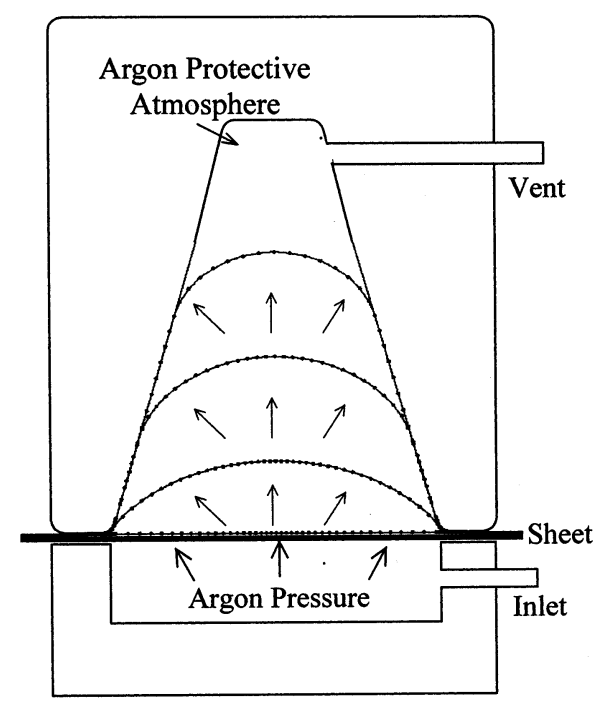

Fig. 2. Schematic diagram for superplastic bulging of a cone.

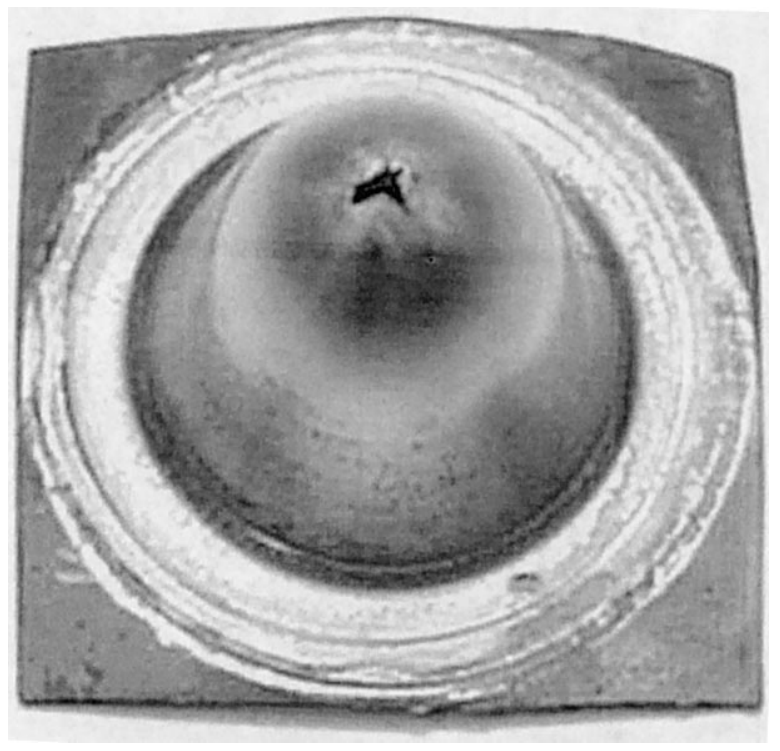

Fig. 3. Fracture bulged part of $\mathrm{Ti}-6 \mathrm{Al}-4 \mathrm{~V}$ sheet.

Table 1

Fracture time and fracture height of $\mathrm{Ti}-6 \mathrm{Al}-4 \mathrm{~V}$ sheets bulged at $900^{\circ} \mathrm{C}$

\begin{tabular}{lllll}
\hline Forming pressure (MPa) & 2.94 & 2.548 & 2.254 & 1.96 \\
Fracture time (s) & 591 & 748 & 883 & 1133 \\
Fracture height (mm) & 45.4 & 48.1 & 49.0 & 51.1 \\
\hline
\end{tabular}

fracturing eventually occurs. It has been shown that there is little opportunity to delay the onset of strain concentration due to non-uniform stress states, but that subsequent flow localization can be limited by control of the post-uniform deformation process [12].

By applying the flow localization factor for analysis, prediction of instability, quantitative description of strain concentration beyond instability, and thus control of the post-uniform deformation process can all be achieved. Achieving this requires a series of experiments and the aid of finite element simulations.

\section{Superplastic fracture bulging experiments}

\subsection{Experimental details}

The 'flow localization factor' can be the index of strain concentration; however, the relation between this parameter and the forming limits of superplastic materials requires further investigation through experimentation. Thus, we designed a series of experiments of superplastic fracture bulging, a design accomplished by placing a $2.0 \mathrm{~mm}$ thick $\mathrm{Ti}-6 \mathrm{Al}-4 \mathrm{~V}$ sheet over a coneshaped die and blowing high-purity argon gas over the sheet at $900^{\circ} \mathrm{C}$ with constant pressures of $2.94,2.548$, 2.254 , and $1.96 \mathrm{MPa}$ until fracturing occurs. The flow localization factors at fracture points are also computed by means of finite element simulations. Since the top of the cone-shaped part is subjected to the largest amounts of deformation, fracturing will happen at that point.

Fig. 2 shows the schematic diagram for the superplastic bulging of a cone. The die entry radius of the upper die is $2 \mathrm{~mm}$, and the hollow part of it is conical with a cone angle of $14^{\circ}$ so that the formed part can be taken out more easily. Gas is blown into the lower die with a rim around the edge in order to clamp the softened material at the high temperature and thus enhance the sealing effect.

\subsection{Experimental results}

After the fracture bulging experiments are accomplished, the fracture heights are measured; the typical formed part is shown in Fig. 3. The fracture time (the time required to fracture) and fracture height for various forming pressures are shown in Table 1 (the data presented are average values of several experiments). The experimental results show that lower forming pressures lead to longer fracture times and greater forming heights.

\section{Finite element analysis}

\subsection{Basic assumptions and mesh}

A commercial finite-element package, ABAQUS, is used to perform the modeling and analysis for the constant pressure bulging experiments. The material properties and the process of deformation are very complicated in real cases; thus, it requires some reasonable assumptions to simplify the analysis. Although 
superplastic deformation of titanium alloys can exhibit slight to moderate amounts of anisotropy $[13,14]$, the

(a)

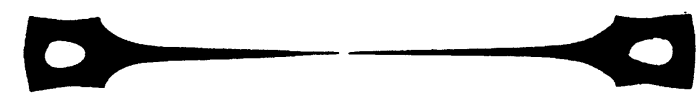

(b)

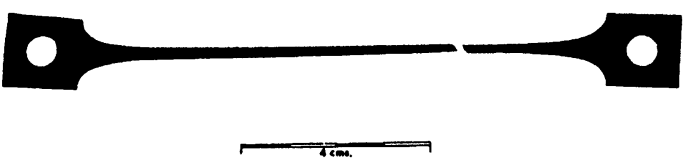

Fig. 4. Shadowgraphs of the fracture in two superplastic alloys (a) unstable plastic flow in $\mathrm{Ti}-6 \mathrm{Al}-4 \mathrm{~V}$ and (b) pseudo-brittle fracture by cavitation in Supral 220 [5].

Fig. 5. Mesh of sheet.

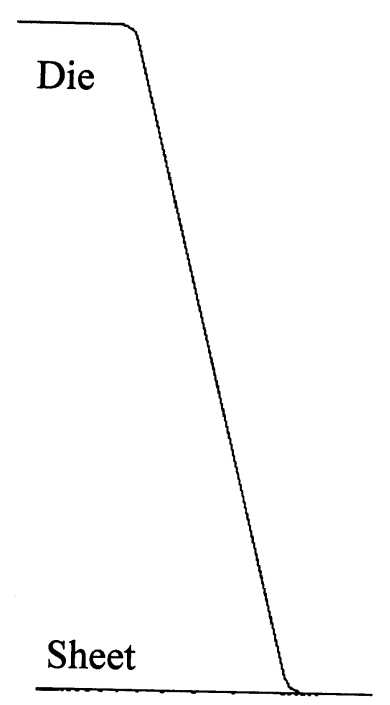

Fig. 6. Sheet and die model for superplastic bulging.

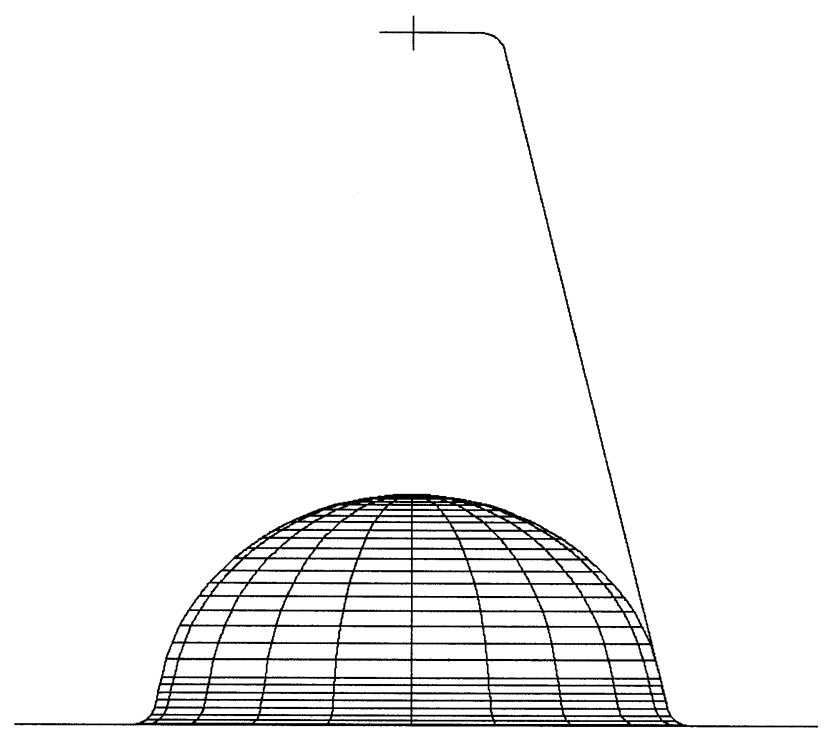

Fig. 7. Deformed mesh of superplastic formed part. majority of the material's resistance to flow localization can be captured with an isotropic flow rule [15]. Zhou et al. [6] have shown that for materials that are less sensitive to cavity growth, the fracture mode is dominated by unstable plastic flow, and unstable plastic flow normally results in the material pulling out to a fine point prior to failure [5]. Fig. 4 [5] shows that the fracture mode of $\mathrm{Ti}-6 \mathrm{Al}-4 \mathrm{~V}$ alloy is dominated by unstable plastic flow; therefore, the void growth can be ignored. All the assumptions are summarized as follows.

1. The sheet material is isotropic, homogeneous, and incompressible.

2. Microstructural weaknesses and variations such as void growth are ignored.

3. The weight of the sheet can be neglected.

4. The forming process is isothermal over the entire specimen.

5. The bending effects of the sheet are negligible; therefore, it can be modeled by membrane elements.

Two-node linear axisymmetric membrane elements are used for the sheet; the mesh is shown in Fig. 5, with the left end being the center of the sheet. The mesh is finer around the center for the largest amounts of deformation. The mesh becomes coarser as the distance from the center increases, but around the die entry region, the mesh is also finer to fit the $2.0 \mathrm{~mm}$ entry radius. The sheet and die model for the superplastic bulging experiments is shown in Fig. 6 .

\subsection{Material properties}

Superplastic deformation is generally regarded as a visco-plastic flow. In order to simplify the complexity of analysis, the behavior of superplastic materials is often characterized by the relation $\sigma=K \dot{\varepsilon}^{m}$; i.e. strain-rate hardening is considered, but strain hardening is not. However, the Ti-6Al-4V alloy possesses a stronger strain hardening effect then other superplastic materials [16]. This is due to the strong dependence of Ti-6Al$4 \mathrm{~V}$ behavior on grain size [17], and it has been shown that grain coarsening occurs during deformation and causes flow hardening [18]. Consequently, the constitutive equation $\sigma=K \dot{\varepsilon}^{m} \varepsilon^{n}$, which is more representative of the Ti-6Al-4V alloy, is used in this study, and the coefficients $m$ and $n$ are taken as functions of strain rate. The material coefficients at $900^{\circ} \mathrm{C}$ are obtained and converted from Refs. [7,16].

\subsection{Verification of the finite element model}

A $413 \mathrm{~s}$ bulging experiment is accomplished by blowing argon gas of $2.205 \mathrm{MPa}$ at $900^{\circ} \mathrm{C}$ to verify the finite element model. The height of the blow-formed part is $20.3 \mathrm{~mm}$, and the simulation result is $20 \mathrm{~mm}$, for which the margin of error is only $1.48 \%$. The deformed mesh 


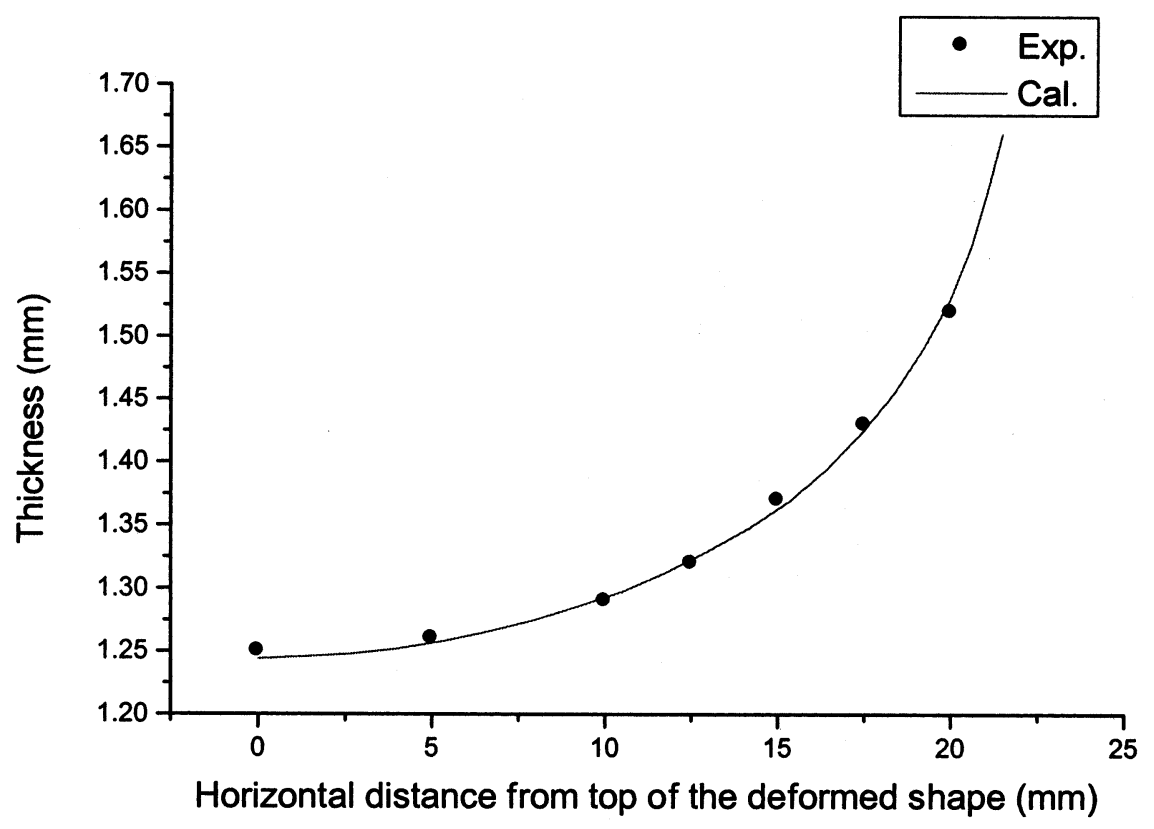

Fig. 8. Thickness distribution of superplastic formed part.

is shown in Fig. 7, and the simulation and experimental thickness distribution of the formed part is shown in Fig. 8. It is clear that satisfactory results can be obtained by applying this finite element model.

\subsection{Simulation results and discussions}

The simulation results for the experiments described in Section 3.1 are summarized in Table 2, where the errors of the forming time are less than $5 \%$, indicating that an effective simulation for superplastic bulging experiments of $\mathrm{Ti}-6 \mathrm{Al}-4 \mathrm{~V}$ sheets can be achieved by applying this finite element model.

The critical point of the observation is the fracture point, i.e. the top of the blow-formed cone, which is in the balanced biaxial condition $\rho=1$. As derived in Section 2.3.2, the flow localization factor in this condition is $\xi_{\mathrm{II}}=\frac{1-2 \gamma}{m}-1$, and it stands for the degree of flow localization. Fig. 9 shows the curves of flow localization factor at the fracture point versus forming time during constant pressure bulging. It is clear that there is an inverse relationship between the forming pressure and the duration of the stable condition, with lower pressures leading to a longer stability.

According to the curves, the localization process can be divided into three stages:

1. The developing period of initial localized flow: Once the deformation becomes unstable, the flow localization factor somehow grows at a higher rate, with a noticeable increase in the growth rate as the forming pressure increases. This, however, is only a short duration in the entire forming process, and the second stage appears soon after.
2. The steady stage of strain concentration: In this stage, the flow localization factor grows slowly and steadily, and thus the slopes of the curves in Fig. 9 are nearly constant. This stage is the major part of the forming process.

3. The accelerating stage of strain concentration: The formed part approaches its forming limit in this stage, where the flow localization factor again grows fast, and strain concentration is apparent throughout the stage until the end of the forming process, when fracturing occurs.

According to the above observations, an effective superplastic forming process can be designed by controlling the bulging pressure to keep it within the second stage in order to avoid the third stage.

\section{Conclusions}

The relation between superplastic failure behavior and strain concentration is studied via the analysis of instability and flow localization, as well as the finite element simulations for superplastic bulging experiments. Since SPF is a slow process, there is a strong

\section{Table 2}

Simulation results of Ti-6Al-4V sheets bulged at $900^{\circ} \mathrm{C}$

\begin{tabular}{lllll}
\hline Forming pressure (MPa) & 2.94 & 2.548 & 2.254 & 1.96 \\
Fracture height (mm) & 45.4 & 48.1 & 49.0 & 51.1 \\
Fracture time (s) & 591 & 748 & 883 & 1133 \\
$\begin{array}{l}\text { Simulation time to reach the } \\
\quad \text { fracture height (s) }\end{array}$ & 607 & 775 & 921 & 1175 \\
$\quad$ Err $(\%)$ & 2.7 & 3.6 & 4.3 & 3.7 \\
\hline
\end{tabular}




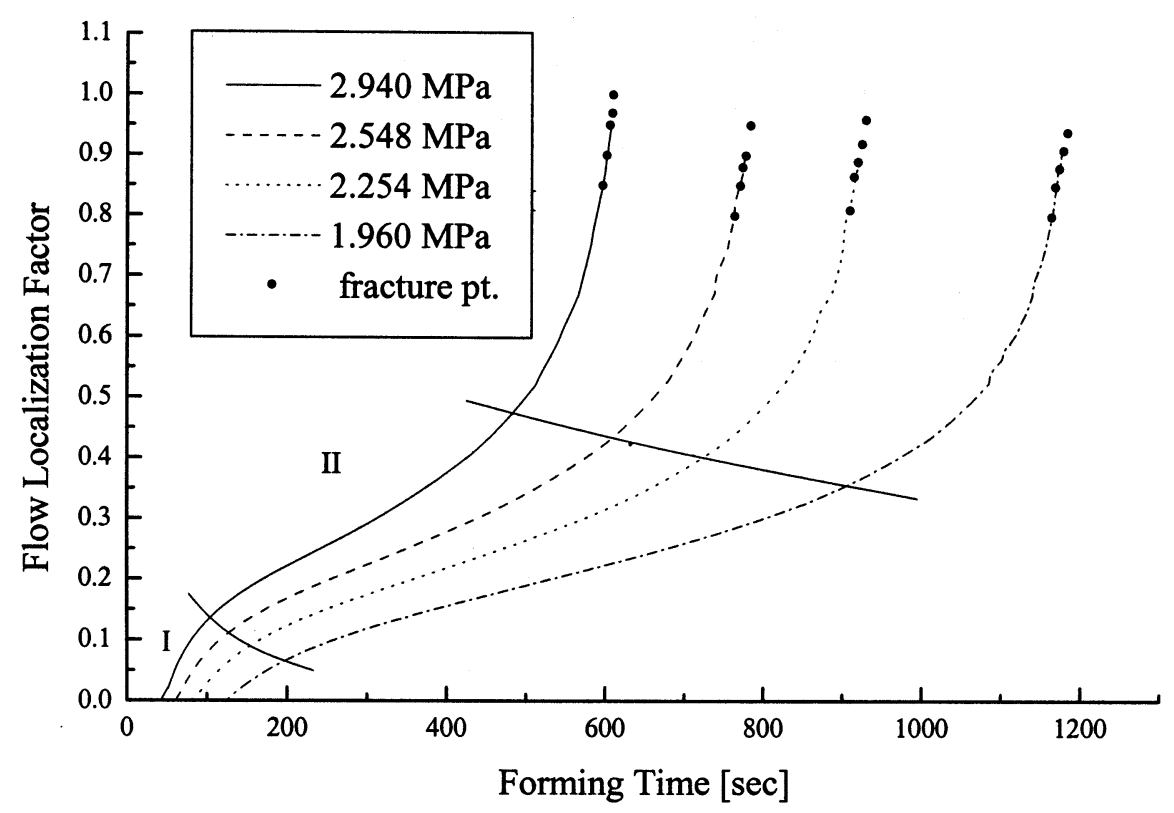

Fig. 9. Flow localization factor vs. forming time.

incentive to increase the efficiency of production. The goal is to form the workpiece in the shortest time while maintaining the required quality; techniques to achieve this goal can be developed by exploring new control schemes [7]. It requires, however, an effective criterion for the evaluation and design of the forming parameters. The quantitative analysis of the localization process of unstable plastic flow proposed in this paper can be applied as an aid in this work, to which we will devote ourselves in the future.

From the analysis and experimental results, we conclude the following.

1. Hart's stability criterion is generalized in terms of strain and strain rate, and the flow localization factor is defined according to the generalization.

2. The proposed 'flow localization factor' stands for the degree of flow localization, and it can be applied in order to quantify the localization process of unstable plastic flow for both uniaxial tension and biaxial stretching.

3. The simulation results, which are in good agreement with experiments of superplastic bulging, are achieved by using the material model that takes into account both strain hardening and strain-rate hardening.

4. The localization process of unstable plastic flow during constant pressure bulging can be divided into three stages, which are the developing period of initial localized flow, the steady stage of strain concentration, and the accelerating stage of strain concentration. The second stage takes the most of the forming time, and the fracturing occurs in the third stage.
5. The presented quantitative analysis of the flow localization process can be applied to develop SPF processes that achieve both high productivity and high quality.

\section{Acknowledgements}

This research was sponsored by the National Science Council of the Republic of China.

\section{References}

[1] E.W. Hart, Acta Metall. 15 (1967) 351-355.

[2] A.R. Tayupov, Scr. Metall. Mater. 30 (11) (1994) 1387-1389.

[3] N.Q. Chinh, G. Kapovics, P. Szommer, I. Kovacs, Mater. Sci. Forum 217-222 (1996) 1455-1460.

[4] E. Sato, K. Kuribayashi, ISIJ Int. 33 (8) (1993) 825-832.

[5] J. Pilling, N. Ridley, in: Superplasticity in Crystalline Solids, Pub. Institute of Metals, London, UK, 1989, pp. 102-158.

[6] D.-J. Zhou, J. Lian, M. Suery, Mater. Sci. Technol. 4 (1988) $348-353$.

[7] X.D. Ding, H.M. Zbib, C.H. Hamilton, A.E. Bayoumi, J. Mater. Eng. Perform. 4 (4) (1995) 474-485.

[8] X.D. Ding, H.M. Zbib, C.H. Hamilton, A.E. Bayoumi, J. Eng. Mater. Technol. Trans. ASME 119 (1997) 26-31.

[9] Du Zhixiao, Li Miaoquan, Liu Mabao, Wu Shichun, Appl. Math. Mech. (English edition) 17 (2) (1996) 133-138.

[10] W.A. Spizig, R.E. Smelser, O. Richmond, Acta Metall. 36 (5) (1988) 1201-1211.

[11] C.H. Cáceres, D.S. Wilkinson, Acta Metall. 32 (3) (1984) 415422.

[12] C.H. Johnson, C.H. Hamilton, H.M. Zbib and S.K. Richter, in: N. Chandra et al. (Eds.), Advances in Superplasticity and Superplastic Forming, The Minerals, Metals and Materials Society, Warrendale, PA, USA, 1993, pp. 3-15. 
[13] P.G. Partridge, D.S. McDarmaid, A.W. Bowen, Acta Metall. 33 (4) (1985) 571-577.

[14] D.A. Mosher, P.R. Dawson, Mech. Mater. 19 (1994) 59-72.

[15] D.A. Mosher, P.R. Dawson, J. Eng. Mater. Technol. Trans. ASME 118 (1996) 162-168.

[16] C.J. Lin, Principles and Applications of Superplastic Metal
Forming, first ed., Pub. Aero-industry, Beijing, 1990, pp. 51-52 in Chinese.

[17] D. Lee, W.A. Backofen, Trans. TMS-AIME 239 (1967) 10341040.

[18] A.K. Ghosh, C.H. Hamilton, Metall. Trans. 13A (1982) 733741. 